\title{
Das k.u.k. militärgeographische Institut
}

\author{
Franz Almer ${ }^{1}$ \\ Technische Universität, Graz
}

\begin{abstract}
The Institue of Military Geograph was an important institution. founded at the begining of the $19^{\text {th }}$ century (the exactly date is unknown). In Milan (today Italy) was the central institution for the whole Austrian monachy, where the newest topological maps were kept. The succesor of the institute was the Austrian institution of the "Bundesamt für Eich- und Vermessungswesen.
\end{abstract}

\section{Vorgeschichte}

Kaiserin Maria Theresia (1717 - 1780) wurde als Tochter des unerwartet früh verstorbenen Vaters, Kaiser Kar1 VI. zur Nachfolgerin ernannt. Die Erziehungsverantwortlichen bei Hof besorgten ihr als Hauslehrer Professor Johann Jakob Marinoni (geb. 1676 in Udine, gest. 1755 in Wien). Dieser weckte bei Maria Theresia großes Interesse für die naturwissenschaftlichen Fächer: Mathematik, Geographie, Astronomie usw. Johann Jakob Marinoni, Sohn wohlhabender Eltern kam 1696 nach Wien. Er wurde auf Grund seiner Vorstudien und nach zweijährigem Besuch im Alter von 22 Jahren an der Wiener Universität zum Doktor der Philosophie promoviert. Er widmete sich der praktischen Mathematik, erteilte Mathematik-Unterricht, unter anderem auch den Söhnen und Töchtern der kaiserlichen Familie. 1703 (27-jährig) wurde er mit dem Titel eines kaiserlichen Mathematikers ausgezeichnet. Marinoni wurde durch geodätische und ingenieurtechnische Arbeiten immer bekannter und erstattete in den Jahren 1710 - 1711 mit seinem väterlichen Freunde, dem Ingenieur - Hauptmann Graf Leander Anguissola (1653 - 1720) Vorschläge für die Errichtung einer Ingenieur - Akademie zur Heranbildung von militärischen und zivilen Ingenieuren, die Kaiser Karl VI. mit Patent vom 24.XII.1717 bewilligte.

\footnotetext{
${ }^{1}$ Hofrat Honorar Prof. Franz Almer ${ }^{\dagger}$, Ehrensenator der TU Graz, starb im Alter von 92 Jahren $(* 3$. November $1916-\dagger 31$. Oktober 2008)
} 
Anguissola und Marinoni wurden zu Leitern dieser Ingenieur-Akademie ernannt, die eine Vorläuferin der technischen Universität war. J.J. Marinoni's Ruhm stieg weit über die Grenzen Österreichs hinaus. Er wurde zum kaiserlichen Rat ernannt und geadelt. Fünf Akademien der Wissenschaften aus Europa zeichneten inn durch seine Mitgliedschaft aus. ${ }^{2}$

In Wien lebte damals als Vorstand des so genannten AstronomischTrigonometrisch Depatements des Generalquatiermeisterstabes Oberst Franz Xaver Richter von Binnenthal (1759-1840). Diese Einrichtung kann als Vorläufer des k.u.k. militärgeographischen Institutes angesehen werden.

Napoleon und andere namhafte Feldherren waren der unabdinglichen Meinung, dass es eine der wichtigsten Voraussetzungen sei, präzise Landkarten zu besitzen, um einen Krieg zu gewinnen. Ein einziges Volk ist bekannt, das ohne kartographische Unterlagen erfolgreiche Kriege führte - das waren die Türken. Ihre Feldzüge gingen über Kontinente hinweg, ihre militärische Planung geschah nur auf Grund mündlicher Aussagen von Häschern und Spionen.

\section{Österreichische Landesaufnahme (1764 - 1787)}

Für diese Landesaufnahme wurden ungefähr 4000 Sektionen im Massstabe 1 : 28800 erzeugt, die das damalige Gebiet Össterreichs umfaßte. Zusätzlich zu jeder Sektion wurde eine wörtliche Beschreibung des zeichnerischen Inhaltes verfasst - eine einzigartige Darstellungsart in Europa. Eine strenge Geheimhaltung des Kartenmaterials (- nur ein Exemplar für den Kaiser -) war nicht durch eine fehlende Vervielfältigungsmöglichkeit gegeben, sondern nur, um allfälliger Spionage vorzubeugen.

\section{4/1815 Wiener Kongress}

Vereinfacht gesagt, wurden die verschiedenen napoleonischen Feldzüge und Eroberungen wieder teilweise rückgängig gemacht. Unter anderem wurde die Österreichische k.u.kMonarchie mit der Verwaltung von Venetien und Lombardei im Pariser Frieden (30. Mai 1814) beauftragt. Die österreichischen Offiziere lernten im Zentrum von Mailand das "Militärgeographische Institut" kennen. Die damalige Bezeichnung war "Deposito della Guerra". Das Gründungsdatum dieser Institution ist trotz sorgfältiger Nachforschungen bis heute unbekannt geblieben.

Der im österreichischen Hauptquartier in Mailand anwesende Chef, Feldmarschall -Leutnant Franz Xaver Richter von Binnenthal (1759 - 18401,

\footnotetext{
${ }^{2}$ Die Gründung der Österreichischen Akademie der Wissenschaften erfolgte erst $1847 / 48$ in Wien - eine der letzten in Europa.
} 
früher Vorstand des so genannten Astronomisch - Trigonometrischen Departementes des General-Quartiermeisterstabes in Wien, erhielt den Auftrag, dieses Mailänder Institut (Deposito della Guerra) zu besichtigen und einen ausführlichen Bericht an den Kaiser in Wien zu erstatten. Anekdotenhaft soll sich der Kaiser in Wien geäussert haben: "Ja, darf denn das sein, dass eine so wichtige kartographische Einrichtung in Mailand und nicht in Wien ist?"

Nach Anordnung des General-Inspektors des Geniewesens sollte dieses Mailänder Institut Karten, Pläne und andere verwandte topographische Arbeiten sammeln und aufbewahren, damit im Erstfalle die nötigen Planungsunterlagen bereit seien. Auch wurde der Stand der topographischen Unterlagen festgehalten: Geplante Vergrösserungen einzelner Institutsteile, kartographische Arbeiten, die bereits beendet waren, welche noch im Werden waren, welche noch zu ergänzen waren usw. Welche Methoden bei den Triangulierungen, bei den Detailaufnahmen, beim Kupferstich usw. beobachtet worden sind.

Ein weiterer Bericht enthält den Stand des Personales, der aus Militaristen und Zivilisten zusammengesetzt war; ebenso sind die vortrefflichen Vermessungsinstrumente, Theodolite, Basismesseinrichtungen, Messtische uam. in aufgeschlüsselter Form, ebenso dem Maschinenpark für Gravur und Druck.

Der Direktor dieses Militärgeographischen Institutes war Major Anton Campana, geboren 1776 in Portici bei Neapel. Er war Kommandeur des herzoglichen Parmaischen Constantinischen Sankt Georgsordens, Ritter des königlichen sardischen Sankt Mauritius- und Lazarus- Ordens. Aus dem Auszug von Major Heinrich HARTL's MGI sei zitiert - Mitteilungen, Jahrgang 1888:

"Major Anton Campana ist ein Mann, der mit allen zu diesem Geschäft nötigen Kenntnissen ausgerüstet ist, und mit Leidenschaft, ohne die in diesem Fache nichts Vollkommenes geleistet wird, arbeitet. Es wäre daher schade, wenn dieses Institut aufgelöst, oder dessen Direktor davon entfernt würde, denn nur er allein kann es wissen, welchen Grad von Richtigkeit die bisher erschienenen Arbeiten dieses Institutes haben, und welchen Mängeln abzuhelfen ist."

Ein weiterer Auszug: Mailand, am 13. Juli 1814. Richter von Binnenthal, FMLT. $\mathrm{m} / \mathrm{p}$.

"Derzeit sind mit Einbeziehung des Direktors 17 Offiziere und 29 Zivilpersonen angestellt. Ihre Gehälter betragen $47787 \mathrm{fl} 18 \mathrm{kr}$. Das Material an Papier, Farben, Federn, Bleistifte usw. dürften nach Angabe von Major Campana jährlich $9300 \mathrm{fl}$ zu stehen kommen, so dass also das ganze Institut einen Kostenaufwand von ugf. $58000 \mathrm{fl}$ beträgt."

\section{Splügenpass (italienisch: Passo dello Spluga)}

Bei der Errichtung der innerösterreichischen Institution wurde das umfangreiche mailänder Kartenmaterial über den Splüngenpass transponiert. Dieser Pass ist 
an der italienisch - schweizerischen Grenze, 2113 m über dem Meere, zwischen Chiavenna und Splügen (Hinterrheintal). Dieser Alpenübergang wurde trotz seiner enormen Seehöhe $2113 \mathrm{~m}$ gewählt, weil er geologisch sehr günstig war. Durch das weiche Gesteinsmaterial wurde mit geringer Steigung in vielen Serpentinen der Pass relativ leicht überwunden. Seinerzeit wählte auch Napoleon mehrmals diesen Pass, wenn er vom Süden nach dem Norden zog. Während sich unter Führung von Major Anton Campana der gewaltige Tross von Reitern und schweren bespannten Lastwagen in Richtung Wien bewegte - rümpften Insider in Wien die Nase; Zwei Dinge erregten die Wiener:

1. Major Campana war Stock-Italiener aus Portici bei Neapel, der kein deutsches Wort verstand.

2. Major Campana war ein sehr tüchtiger kartographischer Fachmann und Manager, hatte aber keinen österreichischen Adelstitel, was in der Kaiserstadt Wien für ein solches wichtiges Amt untragbar schien.

Zu Punkt 2.: Diesem Manko konnte abgeholfen werden, indem Major Campana während des Rittes über den Splügenpass nach Wien zum "Ritter von Splügenberg" geadelt wurde.

Zu Punkt:2r: Dieser Umstand erledigte sich in Kürze von selbst, da der inzwischen zum Oberst beförderte Ritter von Splügenberg nach 14 Monaten in Wien starb (1841.02.28).

1839 Übersiedlung des MGI von Mailand nach Wien:

17 Offizier und 29 Zivilpersonen

16000 kg Einrichtungsgegenstände mit Pferdefuhrwerken

22 Tage lange Reise nach Wien.

Es gab Transportschwierigkeiten verursacht durch einen Wettersturz mit dem Zusammenbruch einer Brücke. Die Entfernung betrug 120 Österreichische Meilen = ugf. $910 \mathrm{~km}$ (1 österr. Meile =7,586 km) für die Distanz Mailand - Wien. Mit grossem Eifer wurde das k.u.k. Militärgeographisches Instituts Gebäude in Wien errichtet. Es gibt Schriftstücke, die betonen, dass auch Pioniere beim Neubau mithelfen mussten, um den Fertigstellungstermin einhalten zu können. Die Kommandanten der Pioniereinheiten hatten wenig Freude mit den "Fremdarbeiten" ihrer Truppen.

Am 1. Jänner 1840 Inbetriebnahme des A - Gebäudes (Amtsgebäudes) auf dem später sobenannten Friedrich Schmidtplatz 3 im VIII. Bezirk (Josefstadt). Das Wiener Rathaus war zu dieser Zeit erst in Planung.

Das Personal der neuen Wiener Dienststelle wurde auf das Sechsfache gegenüber Mailand aufgestockt und war in 8 Abteilungen neu gegliedert:

1. Institutsdirektion: Direktor Anton Campana, Generalmajor. (12 Individuen) Unterdirektor: Oberst von Skribanek 
2. Topographische Zeichnungskanzlei

(14 Individuen)

3. Abteilung der Lithographen

(17 Individuen, davon 2 Taubstumme)

4. Abteilung der Kupferstecher

(18 Individuen)

5. Abteilung der Pressen

(22 Individuen)

6. Militärtriangulierungs- und Calcul-Büro

(7 Individuen)

7. Militärische Zeichnungskanzlei des Generalquartiermeister -stabes (13 Individuen)

8. Militär - Mappierungs - Abteilungen

(23 Individuen)

Insgesamt: 126 Individuen, davon 17 aus Mailand übernommen.

Schon 30 Jahre später (1870), nach Errichtung des Amtsgebäudes des k.u.k. Militärgeographischen Institutes in Wien auf dem nachmaligen Friedrich Schmidtplatz, platzte das Gebäude aus allen Nähten. Der Maschinenpark der Kartendruckerei wurde im Zeitalter der rasanten Industrialisierung wesentlich vergrössert, neue Messgeräte und Messinstrumente wurden beschafft. Für die Schaffung der Vermessungsgrundlagen, wie der astronomisch-geodätischen Orts - und Zeitbestim-mung waren neue Instrumente erforderlich, die sorgfältig während der Innendienstzeit überprüft und justiert werden mussten. Es kam 1870 zu einer Aufstockung eines dritten Obergeschosses des A-Gebäudes, dessen Fassade dem heutigen Aussehen entsprach. Zusätzlich wurde auf dem südöstlichen Teil des Daches ein Zeitballon angebracht, der ein optisches Zeitzeichen für die Wiener Bürger abgab. 1936 wurde dieser Zeitballon von der Atomuhr des Eichamtes in Wien ferngesteuert. Durch einen Bombentreffer im Oktober 1944 wurde dieser Zeitballon vernichtet und nicht mehr wiederaufgebaut. 1906 war neuerlich die Raumnot des Amtsgebäudes auf dem Friedrich Schmidtplatz 3 exorbitant fühlbar. Es wurde daher das freigewordene militärische Gebäude auf dem Hamerlingplatz als B - Gebäude eingerichtet. Bisher war dieses B - Gebäude als militärisches Bettengebäude in Verwendung gestanden. Hier wurden Stahlrohrbetten für die Kasernen der ganzen Monarchie erzeugt, gelagert, repariert und ausgeliefert. Im Jahre 2006 fand eine würdige Feier des Bundesamtes für Eich- und Vermessungswesen in Zusammenarbeit mit dem Österreichischen Bundesheer statt: "100 Jahre B - Gebäude". 


\section{4 - 1918 Erster Weltkrieg}

Das Militärgeographische Institut wurde an allen Fronten eingesetzt. Am 10. September 1919 erfolgte der Friedensvertrag von St. Germain mit Österreich.

1921: Gründung des Bundesvermessungsdienstes.

Abspaltung des "Kartographischen Institutes" (vormals: Militärgeographisches Institut) Dieses wird als ein Privatbetrieb des Bundes geführt (wie vergleichsweise die Bundesforste).

1923: Gründung des Bundesamtes für Eich- und Vermessungswesen.

Das k.u.k. Militärgeographisches Institut erlebte im Jahre 1921 ein ganz besonderes unverständliches Schicksal - es wurde aus dem einheitlichen staatlichen Vermessungswesen herausgenommen. Von 1921 bis 1938 wurde die Bezeichnung "Kartographisches Institut (ehemals Militärgeographisches Institut)" eingeführt und zum Privatbetrieb des Bundes erklärt. Dieser Betrieb musste sich selbst durch den Verkauf der nun auf 213 Karten beschränkten österr. Maßblättern erhalten (1: 75000 und deren Folgemaßstäbe). Der Erfolg der Einnahmen betrug aber nur 9 Prozent. 91 Prozent mussten vom Bund jährlich zugeschossen werden! Was also keine glückliche kaufmännische Lösung war! Zudem kam mit 19. Dezember 1924 die Währungskrise mit der Einführung der Schillingwährung als enorme Inflation hinzu.

Vor Beendigung des 1. Weltkrieges gab es schon in einschlägigen Fachblättern wichtige Abhandlungen von Offizieren und Hochschulprofessoren, wie eine Neugliederung des staatlichen Vermessungswesens vorstellbar wäre. Der Wiederaufbau des staatlichen Vermessungswesens nach dem ersten Weltkrieg war sehr schwierig. Grosse Teile des Personals verliessen ihre angestammte Arbeitsstätte und gingen in ihre engere Heimat. Flächenmässig wurde Österreich auf ein Achtel reduziert, nur das neu geschaffene Burgenland zeigte einen kleinen Flächenzuwachs von Ungarn. Wertvolle Archivbestände, Geräte, Vermessungsinstrumente,. photogrammetrische Auswertegeräte (z.B. die genialen automatischen Auswertegeräte nach Eduard Ritter von OREL, im Jahre 1907 erdacht und konstruiert) mussten den Sieger- beziehungsweise den Nachfolgestaaten (Tschechoslowakei, Ungarn, Jugoslawien und Italien) abgegeben werden. Die praktische, beziehungsweise kaufmännische Führung des kartographischen Institutes hatte ein Regierungsrat Kliesch über (ehemaliger Feldwebel des MGI).

1933: Unter Bundeskanzler Dr. Engelbert Dollfuss (1892 - 1934) wurde das demokratische österreichische Parlament ausgeschaltet und die Einparteienregierung, genannt "Vaterländische Front" eingeführt. Bekanntlich wurde Dollfuss im Juli 1934 ermordet. Sein Nachfolger wurde Dr. Kurt von Schuschnigg (1897 - 1977). Dieser war streng autoritär und militärisch ausgerichtet. Er führte 1935 die allgemeine Wehrpflicht ein, ohne auf den Widerstand der 
Westmächte und des Völkerbundes zu stossen. (Stille Duldung der Westmächte). Nach dem Friedensvertrag der Westmächte in St.Germain durfte das "Restösterreich" nur ein Söldnerheer von maximal 30000 Mann unterhalten. Tatsächlich waren es maximal ungefähr 23000 Mann. Dieses Söldnerheer bestand zum Grossteil aus ehemaligen Soldaten der österreichischen Monarchie.

1935: Reg. Rat Kliesch sah nun die Möglichkeit gekommen, das "Kartographische Institut (ehemals Militärgeographische Institut )" dem neu entstandenen Bundesheer einzuverleiben. Damit hätte für Reg. Rat Kliesch und seine Mitarbeiter die Möglichkeit bestanden, wieder militärischen Charakter zu bekommen; dadurch versprach man sich bessere Beförderungschancen. Im Dienst wäre das Tragen der ehemaligen Uniform mit Orden und Ehrenzeichen möglich gewesen. Die Mehrzahl des besagten Personals waren ja ehemalige Soldaten.

Diesbezügliche Verhandlungen mit den Parlamentariern und mit Bundeskanzler Schuschnigg waren bereits aufgenommen. Wie im stenographischen Protokoll des Parlamentes mit Nr. 978 vom 4. Jänner 1935 nachzulesen.

Wutentbrannte handgeschriebene Briefe von Professor Dr. Doleschal (Technische Hochschule Wien) an Oberrat Dipl.-Ing. Karl Lego (Bundesamt für Eich- und Vermessungswesen Wien) durchkreuzten die heimlichen Pläne von Reg. Rat Kliesch. Durch das vehemente Einschreiten von Prof. Dr. Doleschal und OR. Dipl.-Ing. Lego blieb also weiterhin das "Kartographische Institut (ehemals Militärgeographisches Institut)" als Privatbetrieb des Bundes bis zum 13. März 1938 bestehen.

13. März 1938: Einmarsch der Deutschen Truppen in Österreich. Überführung des Österreichischen Bundesheeres

Neugliederung des Österreichischen Vermessungswesens in die Hauptvermessungsabteilung XIV (HV X I V ). Diese war zugehörig dem Reichsinnenministerium. - Damit waren auch die Bestrebungen von Reg. Rat Kliesch endgültig untergegangen.

8. Mai 1945: Ende des zweiten Weltkrieges in Europa. Die Ostmark wird wieder als unabhängige Republik Österreich gewertet. Die vier Besatzungsmächte (Amerika, England, Frankreich und Russland) besetzen nach den Beschlüssen der Konferenz von Jalta (1943) das österreichische Staatsgebiet. (Staatsvertrag 1955). Als erstes Gesetz für das wiedererstandene Österreich wurde das "Wiederherstellungsgesetz" geschaffen, wodurch alle bis zum 13. März 1938 geltenden Gesetze und Verordnungen wieder in Kraft getreten sind. Trotzdem gab es auch da Änderungen gegenüber 1938, die zum Teil auch das staatliche Vermessungswesen betrafen: Bundesgesetzblatt für die Republik Österreich. 27.Stück/Jg.1946, ausgegeben am 12. Juni 1946:

85. Kundmachung der Provisorischen Staatsregierung vom 18. Oktober 1945 über die Aufhebung deutscher Rechtsvorschriften auf dem Gebiete der 
Behördenorganisation (31. Kundmachung über die Aufhebung von Rechtsvorschriften des Deutschen Reiches) :

Weiters: das Gesetz über die Bildung von Hauptvermessungsabteilungen vom 18. März 1938,Deutsches R.G.BI. I S. 277 (G.BI.f.d.L.Ö. Nr. 33/1938)

die Verordnung zur Einführung des Gesetzes über die Neuordnung des Vermessungswesens im Lande Österreich und in den sudetendeutschen Gebieten vom 15. Februar 1939, Deutsches R.G.BI. I S.277 (G.BI.f.d.L.Ö. Nr. 238/39), das Gesetz Über die Neuordnung des Vermessungswesens vom 3. Juli 1934, Deutsches R.G.BI. I S.277 (G.BI.f.d.L.Ö.Nr. 238/1939)

die Verordnung zur Einführung des Beschlussrechtes in den Reichsgauen der Ostmark und im Reichsgau Sudetenland vom 6. Mai 1940, Deutsches R.G.BI. I S.805.

Weiters das Gesetz über. die Vollzugsanweisung der Staatsregierung vom 6. Juli 1919, St.G.BI.Nr. 380,

betreffend einheitliche Regelung des gesamten staatlichen Vermessungswesens, die Verordnung der Bundesregierung vom 21.September 1923, B.G.BI.Nr.550,

über die Auflassung der Normaleichungskommission und die Vereinfachung der Organisation des Eichwesens, die Verordnung des Bundesministeriums für Handel und Verkehr vom 3.Dezember 1923, B.G.BI.Nr. 613, betreffend das Statut des Bundesamtes für Eich- und Vermessungswesen.

Damit war die Vereinheitlichung des staatlichen Vermessungswesens zum Bundesamt für Eich- und Vermessungswesen abgeschlossen.

\section{Literatur}

"Das Kaiserlich - Königliche Militärgeographische Institut zu Mailand (M.G.I.), 1814 - 1839." von Hofrat DI Robert Messner, Wien 1986.

"Materialien zur Geschichte der astronomisch-trigonometrischen Vermessung der österreichisch-ungarischen Monarchie", gesammelt und bearbeitet von Heinrich Hartl, k.k. Major im M.G.I.; veröffentlicht in den Mitteilungen des M.G.I. im Jahre 1888, Seite 144-150 und 171-222.

"75 Jahre Kartographie am Hamerlingplatz, 1905-1980, Bundesamt für Eich- und Vermessungswesen, Landesaufnahme." Von Hofrat DI Robert Messner und weiteren neun Mitarbeitern.

"100 Jahre B-Gebäude des BAfEuV auf dem Hamerlingplatz." CD, 2006.

"Handgeschriebene Briefe von Prof. Dolezal und OR. DI Lego" (Im Privatbesitz des Verfassers). 